Boise State University

ScholarWorks

$1-1-2003$

\title{
Modeling and Design of Polythiophene Gate Electrode ChemFETs for Environmental Pollutant Sensing
}

T. V. Krishna

Boise State University

Jeffrey R. Jessing

Boise State University

Dale D. Russell

Boise State University

Jonathan Scaggs

Boise State University

Lisa R. Warner

Boise State University

See next page for additional authors 
Authors

T. V. Krishna, Jeffrey R. Jessing, Dale D. Russell, Jonathan Scaggs, Lisa R. Warner, and Joe A. Hartman 


\title{
Modeling and Design of Polythiophene Gate Electrode ChemFETs for Environmental Pollutant Sensing
}

\author{
T. Vamsi Krishna, Jeffrey R. Jessing, Dale D. Russell, Jonathan Scaggs, Lisa R. Warner, and \\ Joe A. Hartman \\ Boise State University \\ 1910 University Drive \\ Boise, Idaho 83706.
}

\begin{abstract}
Water-borne pollutants such as volatile organic compounds are a serious environmental concern, which has increased the demand for chemical sensing elements. Solidstate sensors based on catalytic gate devices are a subject of current research, however they are restricted in practical applications because of their inability to operate at room temperature. Conducting polymer FETs, which employ a conducting gate polymer, have received much attention due to their unique electronic and optical properties. Polythiophene is chosen as the semi-conductive gate polymer in this work. A functional group attached to the polythiophene is used to detect analytes (i.e., mercury in this work) of interest. The selectivity of the derivitized polythiophene to mercury can be rationalized based on the size of the ring, presence of oxygen and nitrogen donor atoms. In this paper, the modeling and design of a polythiophene gate electrode ChemFET will be discussed. Specifically the model development and resultant device simulations using Silvaco TCAD will be presented. Using this model various current-voltage characteristics of the ChemFET corresponding to parameters such as substrate doping, gate oxide thickness, various gate stacks, and device geometries are presented.
\end{abstract}

\section{INTRODUCTION}

$\mathrm{W}$ ater-borne pollutants are a serious environmental concern with respect to maintaining a safe ground water supply. Hence, there is high demand for effective and efficient chemical sensing devices that can operate in harsh environments. Traditional techniques involve collecting a sample from the site and analyzing that sample in the laboratory. This approach is time consuming and often leads to data interpretation problems, since the sample is remotely analyzed in a laboratory. To obviate these problems a new category of microfabricated chemical sensors, such as chemiresistors and ChemFETs (chemical field effect transistor) are being investigated [1]. Conducting polymer FETs, which employ either a conducting polymer gate or a conducting polymer channel have received much attention due to their unique electronic properties. In this work, the ChemFET under investigation is of the former configuration, i.e., using the polymer as the gate electrode. The major types of devices in the ChemFET family are the ion selective FET (ISFET), enzyme FET (ENFET), and basic ChemFET [1]. The primary disadvantage of the ENFET and ISFET structure is that they require a large reference electrode which makes them less attractive for integrated, miniaturized sensor applications. However, the basic ChemFET does not require a large reference electrode because the silicon substrate serves as a sufficient reference point in the circuit [1]. ChemFET supporting electronics are relatively simple and can be accomodated on the same silicon substrate as the sensors themselves without appreciably increasing the overall size of the sensing system. These compact, inexpensive sensors can be deployed in the field for real-time detection of a various analytes. In this work we examine mercury.

The effectiveness of a microfabricated chemical sensor is primarily determined by three factors: sensitivity, selectivity, and robustness [1]

Sensitivity is a measure of the lowest level of chemical concentration that can be detected in the sensing environment. In applications, such as ground water pollutant detection, it is highly desirable to know whether certain species of interest has exceeded a safe concentration limit. For effective sensing of a given analyte the sensitivity of a sensor must exceed the set EPA limit established for the analyte of concern. For mercury the EPA mandated limit is $0.002 \mathrm{mg} / \mathrm{l}[2]$.

It is desirable to have a high selectivity for a chemical sensor. Selectivity is a measure of the ability to detect a given analyte in the presence of many contaminate species. Thus, a chemical sensing system can be designed with various types of sensors each with a high degree of selectivity to a known contaminate. In chemical sensing applications selectivity is often tailored and adjusted to the analyte(s) of interest. For this work, we designed the sensor to be highly selective to the detection of mercury while ignoring other analytes present in the ground water. The high selectivity has been achieved by suitably selecting the functional group attached to the polythiophene so that only the analyte of interest gets bounded to the functional group and as a result increases the selectivity of the ChemFET.

For a microfabricated sensor to be practical it must exhibit a high degree of robustness in the environment that it is deployed. Specifically, the sensor must maintain stable, predictable characteristics in the presence of a harsh environment. In our application, the sensor is going to be deployed in ground water. The ChemFET's characteristics must remain unchanged except in the presence of mercury contamination. Further, the ChemFET should be reusable. In other words, the charge deposited on the polymer gate 
electrode should be removable to allow the device to be "reset" for repeated use.

The aim of this paper is the design and modeling of a novel ChemFET for use in the detection of mercury contamination in ground water. The ChemFET under investigation is a solidstate device that resembles a conventional MOSFET (metal oxide semiconductor field effect transistor). The primary difference is replacing the polysilicon gate with a conductive polymer gate (polythiophene) that can chemically bind select species. This binding process results in charge collection on the gate electrode, thus given a measured modulation in the underlying channel conductivity.

From a practical point of view, one of the primary advantages to the ChemFET under investigation is it can be fabricated using standard CMOS processing techniques where the conversion of a standard MOSFET to a chemical sensor requires in theory only the replacement of the gate with a suitable chemically sensitive polymer [1]. Further, the choice of the chemically sensitive material enables tailored, high selectivity.

\section{Polythiophene Gate Electrode}

A derivatized polythiophene is selected as a thin film coating on the FET gate for several practical reasons. This polymer is readily deposited by an electro oxidative method, and forms well-characterized, chemically stable, mechanically robust films. It is a semiconductor and permits flow of electrons when biased. It does not swell in moist or aqueous environments because the sulfur moiety does not hydrogen bond, as nitrogen atoms do in several other popular organic semiconductors such as polypyrrole and polyaniline. This fact also means that the polymer is insensitive to changes in $\mathrm{pH}$. It also improves the adhesion characteristics of the polymer on most metal substrates, because the binding site sulfur is not protonated in the presence of water or acids. Polythiophene is relatively impervious to degradation by microorganisms and has good abrasion resistance as well as good adhesion to a wide range of microcircuit compatible substrates.

Two methods have been used to attach the polymer to the gate material, and the choice depends on the target analyte for the sensor. The first method is applicable to both metal ion sensors and molecular recognition sensors. The second method is only applicable to the metal ion sensor fabrication [3]. In the first method, a reactive substituent is attached to the monomer thiophene in the 3-position. The position is important because the 2-position is the preferred site of polymer chain growth. Placing the substituent at the 2 position causes chain termination, and is undesirable. The substituent group is then reacted to attach a chelating ring molecule, which is selective for the target metal ion analyte. In the case of molecular recognition sensors, such as the sensor for benzene, reported here, a template molecule that is identical or very similar to the analyte is bonded at one or more points to monomer thiophene units.
For metal ion sensors, the polymer is formed from a combination of thiophene and derivatized thiophene monomer units. The ratio of these two monomers can be varied to achieve different properties of the bulk polymer and different numbers of analyte binding sites on the polymer surface. Electro oxidative polymerization occurs at relatively mild potentials (about $1 \mathrm{v}$ to $1.5 \mathrm{~V}$ ) from a $0.1 \mathrm{M}$ solution of the mixed monomers. An electrolyte salt is optional in the deposition solution, as deposition will occur without a salt present. When a salt is used, it is $0.1 \mathrm{M}$-tetrabutylammonium hexafluorophosphate, (T.B.A.H.F.P). Film deposition is dependant on the cell geometry, solvent, potential, and substrate.

A molecular recognition sensor has also been developed for benzene. Molecular recognition sites are built into the bulk polythiophene film using a template molecule that has shape, size and electrostatics similar to benzene. The sequence is to attach monomer units to the template molecule using reversible, equilibrium chemistry, then copolymerize the template molecule with thiophene monomers, into a bulk polymer. The template molecules are then removed from the bulk polymer leaving vacancies in the film that will accommodate benzene in terms of both geometry and electrostatics. This electrode has been demonstrated to detect benzene, and exhibits increasing cyclic voltammetric current with increasing benzene concentration.

\section{Modeling of THE ChemFET}

The primary objective for the modeling of the ChemFET is to provide a framework on which actual devices can be fabricated. Silvaco's device (Atlas) and process (Athena) modeling tools were used in this work [4]. Accurate simulation of a ChemFET using these software tools requires the modeling of polythiophene as a gate material. Due to the novelty of this material, only estimates of its inherent electrical properties were used. A diagram of a twodimensional simulated ChemFET is shown in Fig. 1. Variations in device parameters were studied, including gate oxide thickness, polythiophene gate stack composition, physical channel length, and substrate doping. The corresponding threshold voltage and drain current response to varying charge densities incorporated in the gate electrode were computed. In this manner, a first order prediction of the response of the ChemFET to the exposure of mercury could be determined. For instance, on a $20 \mu \mathrm{m} \times 220 \mu \mathrm{m}$ polythiophene gate electrode there would be roughly $15000-$ 60000 binding sites available to capture the analyte of interest. Correspondingly, the charge density that can be deposited on the gate would be between $6.8 \times 10^{8} / \mathrm{cm}^{2}$ to $27.26 \times 10^{8} / \mathrm{cm}^{2}$.

For all the simulations, the source and body were maintained at ground potential, while the gate and drain were fixed at $-5 \mathrm{~V}$. The methodology was to first model a baseline device without any bound charge, thus simulating an unexposed ChemFET. Both drain current $\left(\mathrm{I}_{\mathrm{D}}\right)$ vs. drain 
voltage $\left(V_{D}\right)$ and drain current vs. gate voltage $\left(V_{G}\right)$ curves were generated. Then, a predetermined amount of charge was incorporated into the gate electrode at the gate oxide interface. As the charge density increases, the simulation of increasing exposure to the analyte is achieved. Under each new charge density condition an additional $\mathrm{D}_{\mathrm{D}}-\mathrm{V}_{\mathrm{D}}$ curve was computed. An assumption for the simulations was that the ChemFET was under equilibrium and thus all transient behavior was neglected. A representative simulated $I_{D}-V_{D}$ curve is shown in Fig. 2.

\section{DiSCUSSION}

To a first order, the current response of the ChemFET in the saturation region was modeled using the long channel MOSFET equation:

$$
I_{D S}=\frac{\mu C_{O X}}{2} \frac{W}{L}\left(V_{G S}-V_{T}\right)^{2}
$$

where $I_{D S}, \mu, C_{O X}, V_{T}, V_{G S}, W, L$ are drain current, mobility, oxide capacitance, threshold voltage, gate-to-source voltage, width and length of the channel, respectively. Further, the long channel ChemFET threshold voltage can be expressed as

$$
V_{T}=-\left(\phi_{m s}-\frac{Q_{f}}{C_{O X}}\right)+2 \psi_{B}+\frac{\sqrt{4 \varepsilon_{s} q N_{A} \psi_{B}}}{C_{O X}}
$$

where $Q_{f}, ?_{B}, e_{s}, q, N_{A}$ are interface charge, surface potential, permittivity of silicon, electron charge, and substrate doping concentration, respectively. By extending the conventional MOSFET theory to the ChemFET, it can be shown that the deposition of the charge primarily affects the threshold voltage and hence the drive current of the device. Thus when the polymer detects a mercury ion, the result is a change in threshold voltage, which was measured as modulation of the drain current. Therefore, an observed change in the drain current is a direct consequence of the modification of the work function of the polythiophene gate (due to the accumulation of charge on the gate), which manifests itself as a change in the threshold voltage [5]. To analyze the response of the ChemFET we can show using (1) that the drain current is proportional to the change in threshold voltage as

$$
I_{D S}+\Delta I_{D S}=\frac{\mu C_{O X}}{2} \frac{W}{L}-\left(V_{G S}-\left(V_{T}+\Delta V_{T}\right)\right)^{2}
$$

To the first order (3) can be simplified to $\Delta I_{D S} \propto \Delta V_{T}$. This modulation in the drain current can be quantified in terms of the number of mercury ions that are deposited on the gate of the ChemFET. The response of the ChemFET for various substrate doping levels is shown in the Fig. 3. It can be observed that drain current and its change when charge is added onto the gate is maximum when the substrate doping is minimum, which is due to the fact that it takes less charge on the gate to invert the channel. When gate oxide thickness is varied, keeping all the other parameters constant, from (1) there will be a decrease in the drain current. We can observe the same effect in our simulation results. Fig. 4. shows response of ChemFET as a function of various gate oxide thicknesses. Drain current is maximum when the gate oxide thickness is minimum as can be seen from (1). Normalized drain current as function of gate oxide thickness is plotted in Fig. 5. We can see that for a given gate oxide thickness the modulation of drain current is maximum when all the binding sites are occupied with analyte of interest. Also the modulation is maximum when the gate oxide thickness is more because change in the gate voltage is inversely proportional to the oxide capacitance.

Again from (1) it can be deduced that the drain current is inversely proportional to channel length. Fig. 6. shows variation of drain current as a function of channel length. While Fig. 6. shows the drain current as a function of channel length, Fig. 7. shows the modulation of drain current as a function of channel length (i.e. as the binding sites gets occupied over time). It can be observed that the modulation is maximum when a charge of $2.726 \times 10^{9} / \mathrm{cm}^{2}$ is deposited on the gate, which corresponds to the maximum number of binding sites available on the gate.

\section{FUTURE WORK}

A mask has been designed at Boise State University in order to fabricate the ChemFETs. ChemFETs of various aspect ratios have been incorporated onto the mask along with the test structures to study and quantify the response of the ChemFET. Work is under progress in fabricating and characterizing the devices.

\section{ACKNOWLEDGMENT}

The authors wish to acknowledge the financial support provided by the Environmental Protection Agency, Multipurpose Sensors to Detect and Analyze Environmental Contaminants contract (contract number X-97031101-0).

\section{REFERENCES}

[1] Denise M. Wilson, Sean Hoyt, Jiri Janata, Karl Booksh and Louis Obando, "Chemical sensors for Portable, Handheld Field Instruments," IEEE Sensors Joumal, vol. I,4, 2001, pp. 256-274

[2] http://www.epa.gov/safewater/mcl.html.

[3] Borje Sellergren and Lars Andersson "Molecular Recognition in Macroporous Polymers Prepared by a Substrate Analogue Imprinting Strategy." Journal of Organic Chemistry, vol. 55, 1990, pp. 3381-3383.

[4] Athena and Atlas User's Manuals, Silvaco International, Feburary 2000

[5] J.V. Hatfield, J.A. Covington, J.W. Gardner, "GasFETs incorporating conducting polymers as gate materials", Sensors and Actuators, B 65, 2000, pp. 253-256. 


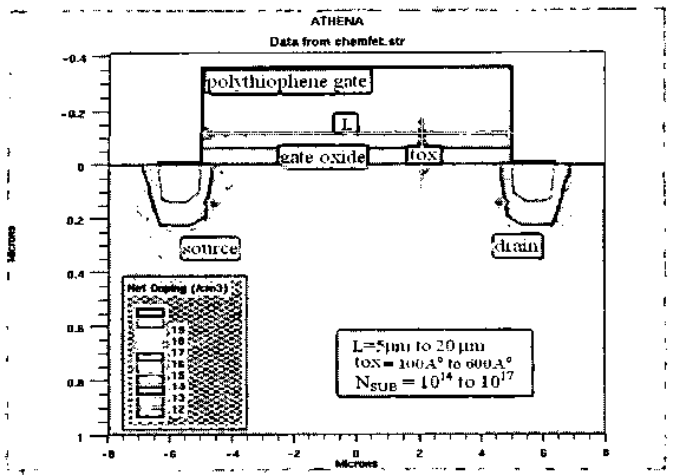

Fig. 1. Simulated ChemFET model in Silvaco.

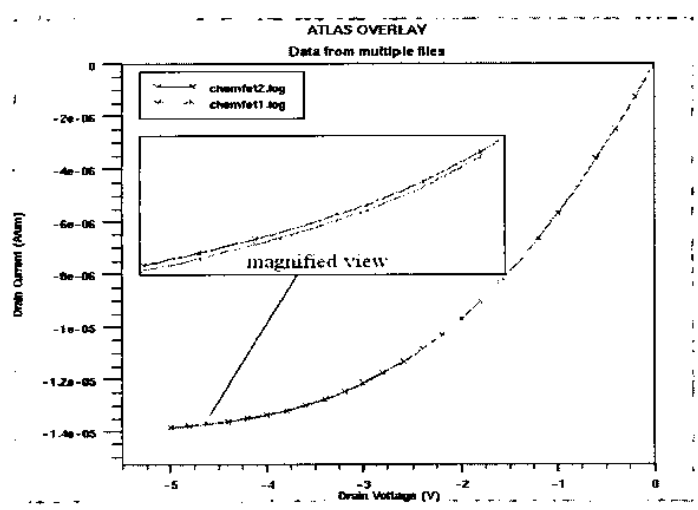

Fig. 2. Representative simulated $b-V_{D}$ curve for a p-channel ChemFET. (tox $=600$ ? , $\mathrm{L}=10 \mathrm{\mu m} \mathrm{N}_{\mathrm{SuB}}=10^{15} / \mathrm{cm}^{3}$ )

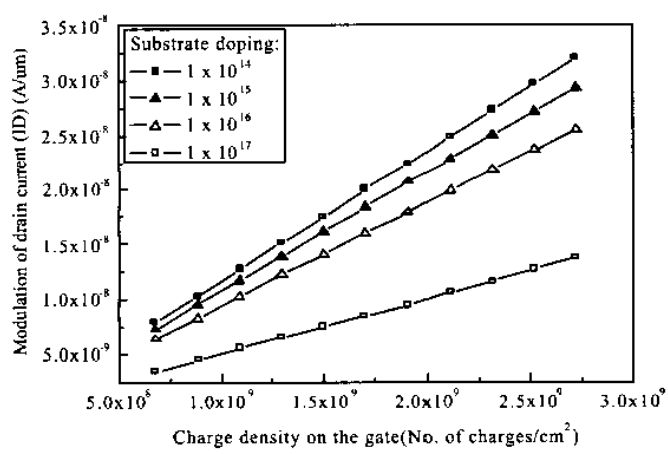

Fig. 3. Modulation of drain current as a function of charge density. (tox $=200$ ? .L=10 $\mathrm{\mu m}$ )

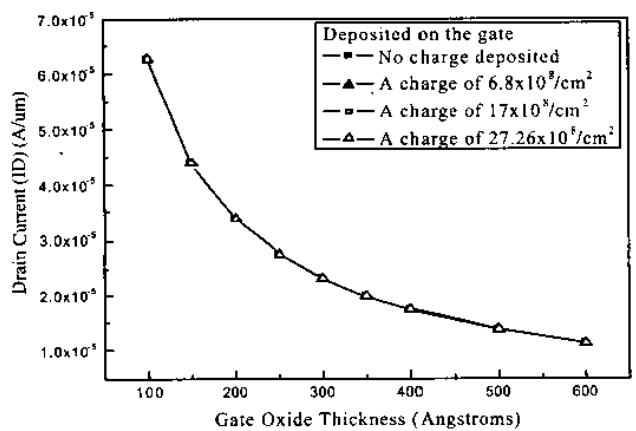

Fig. 4. Drain current as a function of gate oxide thickness. Note at this scale the modulation is not readily apparent. $\left(\mathrm{L}=10 \mu \mathrm{m}, \mathrm{N}_{\mathrm{SUB}}=10^{15} / \mathrm{cm}^{3}\right)$

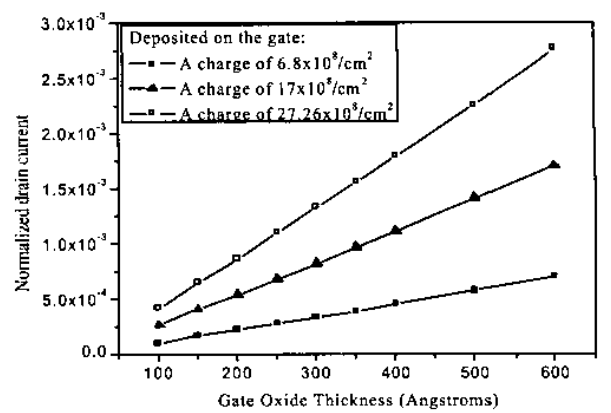

Fig. 5. Normalized drain current as a function of Gate Oxide Thickness. $\left(\mathrm{L}=10 \mu \mathrm{m}, \mathrm{N}_{\mathrm{SUB}}=10^{15} / \mathrm{cm}^{3}\right)$

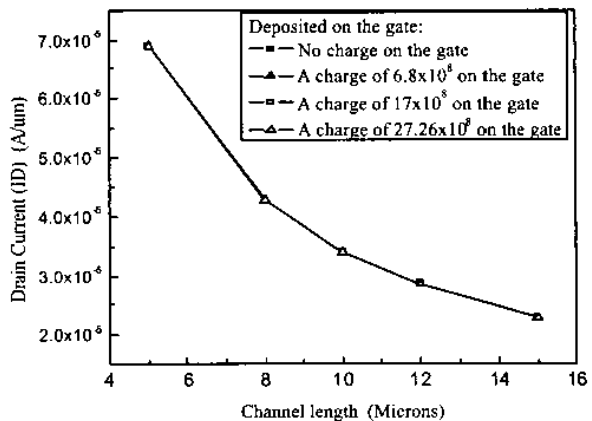

Fig. 6. Drain current as a function of channel length. Note at this scale the modulation is not readily apparent. (tox $=200$ ?, $\mathrm{N}_{\mathrm{SuB}}=10^{15} / \mathrm{cm}^{3}$ )

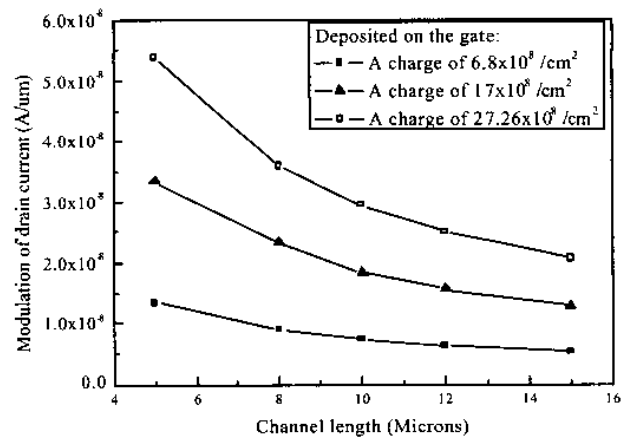

Fig. 7. Modulation of drain current as a function of channel length. (tox $=200 ?, \mathrm{~N}_{\mathrm{SuB}}=10^{15} / \mathrm{cm}^{3}$ ) 